Proc. of the 13th Symposium of Magnetic Measurements and Modeling SMMM'2018, Wieliczka, Poland, October 8-10, 2018

\title{
Magnetic Properties of LaFeCoSi Ring Sample in Low-Frequency Magnetic Field
}

\author{
R. Gozdur ${ }^{a, *}$, P. GęBAra ${ }^{b}$ And K. Chwastek ${ }^{c}$ \\ ${ }^{a}$ Lodz University of Technology, Department of Semiconductor and Optoelectronics Devices, \\ Wolczanska 211/215, 90-924 Lodz, Poland \\ ${ }^{b}$ Częstochowa University of Technology, Institute of Physics, Al. Armii Krajowej 19, 42-200 Częstochowa, Poland \\ ${ }^{c}$ Częstochowa University of Technology, Faculty of Electrical Engineering, \\ A1. Armii Krajowej 17, 42-200 Częstochowa, Poland
}

\begin{abstract}
The paper presents magnetic studies of a ring sample made of LaFeCoSi bulk alloy. Magnetic behavior of the sample was investigated in the temperature range covering the Curie temperature of $306 \mathrm{~K}$. Influence of frequency, temperature, and magnetic saturation on dynamic hysteresis loops and magnetic power losses was determined. Magnetic losses separation into components showed that the hysteresis loss component was dominant in the frequency range up to about $1 \mathrm{~Hz}$. The decline of power losses caused by the temperature changes followed similarly like magnetic polarization changes close to the Curie point of LaFeCoSi alloy.
\end{abstract}

DOI: 10.12693/APhysPolA.136.689

PACS/topics: 75.30.Sg, 75.60.-d, 75.60.Ej

\section{Introduction}

The magnetocaloric effect (MCE) enables one for heating or cooling in active magnetic regenerators (AMR) under the change of external magnetic field $[1,2]$. Nowadays, MCE is the most efficient cooling method and its theoretical efficiency reaches even $60 \%$ [3].

Since 1997 intensive studies of the MCE have started due to discovery of giant magnetocaloric effect (GMCE) in the vicinity of room temperature by Pecharsky and Gschneidner Jr. in the $\mathrm{Gd}_{5} \mathrm{Si}_{2} \mathrm{Ge}_{2}$ alloy [4]. Such excellent magnetocaloric properties are caused by two factors: a second order phase transition from ferro- to paramagnetic state accompanied by the structural first order phase transition close to the Curie temperature $\left(T_{\mathrm{C}}=\right.$ $276 \mathrm{~K})$. However, Gd-based alloys are very costly due to Gd contents and very restricted manufacturing technology. In order to find more economical AMRs, many laboratories have carried studies on MnAs [5], the Heusler alloys [6], or amorphous Fe-based [7] and Co-based [8] alloys. An interesting material, in the Fe-based group of alloys, is $\mathrm{La}(\mathrm{Fe}, \mathrm{Si})_{13}$-type alloy. Relatively high content of $\mathrm{Fe}$ reaching up to 78 at.\% and promising magnetic properties stimulated intensive research on this group of alloys. This type of alloys crystallizes in fcc pseudobinary $\mathrm{La}(\mathrm{Fe}, \mathrm{Si})_{13}$-type phase, whose prototype is $\mathrm{NaZn}_{13}$ structure [9]. The magnitude of MCE in this type of alloys is strongly dependent on chemical composition [10-12], manufacturing technique $[13,14]$, and magnetic excitation [5, 15]. Strong alternating magnetic field triggers the magnetothermal cycle and simultaneously causes magnetic power losses which have an impact on final

*corresponding author; e-mail: gozdur@p.lodz.pl efficiency [5]. A detailed study on the efficiency of magnetocaloric cooling systems should include the hysteresis loss component. An experimental study of LaFeCoSi magnetocaloric bulk alloy close to phase transition and for low-frequency magnetic fields gives better insights into estimation of magnetic power losses under real operating conditions. Moreover, experimental data is a reference to the development and validation of simulation tools based on magnetic hysteresis models $[16,17]$.

\section{Sample and measurements}

The magnetocaloric sample made as a closed magnetic circuit in the shape of a ring was used for investigations of magnetic parameters and power losses. The overall dimensions of the sample were: $\phi_{\text {outer }}=8.9 \mathrm{~mm}$, $\phi_{\text {inner }}=3.7 \mathrm{~mm}, h=5.8 \mathrm{~mm}$, respectively and the weight of the core was $2.021 \mathrm{~g}$. The solid cast of the sample was made of $\mathrm{LaFe}_{10.92} \mathrm{Co}_{1.08} \mathrm{Si}_{1.2}$ alloy with the Curie point $T_{\mathrm{C}}$ at a temperature of $306 \mathrm{~K}$. Phase composition was examined by the Bruker D8 Advance X-ray diffractometer with $\mathrm{Cu} K_{\alpha}$ radiation and LynxEye detector. The magnetocaloric effect and the Curie point were studied using MPMS-XL Quantum Design 5.

The measurements of the hysteresis loops, magnetic polarization, and magnetic power losses were carried out at the temperature ranging from $290 \mathrm{~K}$ to $320 \mathrm{~K}$ and the magnetic field with frequency from $0.2 \mathrm{~Hz}$ to $50 \mathrm{~Hz}$. The experimental study was carried out in accordance with IEC 60404-6 standard. Specific power losses were measured using the UBM method [18] due to the range of measured power. Signals of magnetic field strength, magnetic polarization, and UBM waveforms were acquired with Digital Signal Analyzer NI PCI-4461. All measurements were done under isothermal conditions with a controlled thermal stability of $0.5 \mathrm{~K}$. 


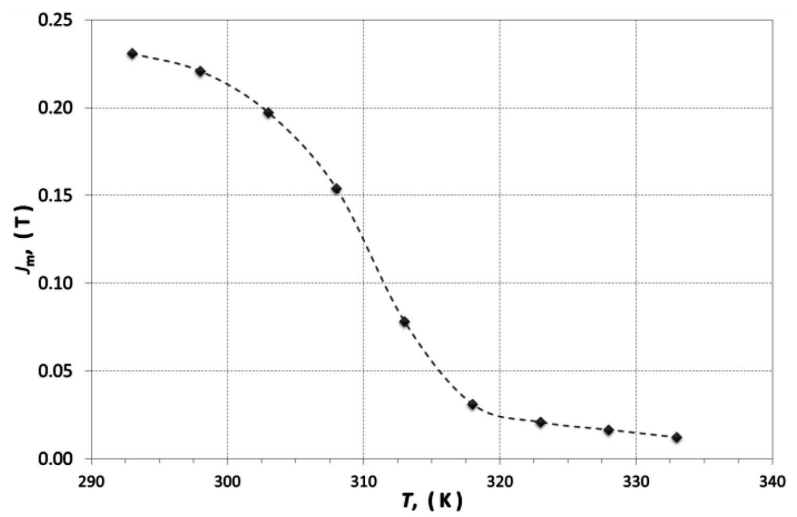

Fig. 1. Influence of temperature and magnetic phase transition on magnetic polarization $J_{m}(T)$ in the bulk sample.

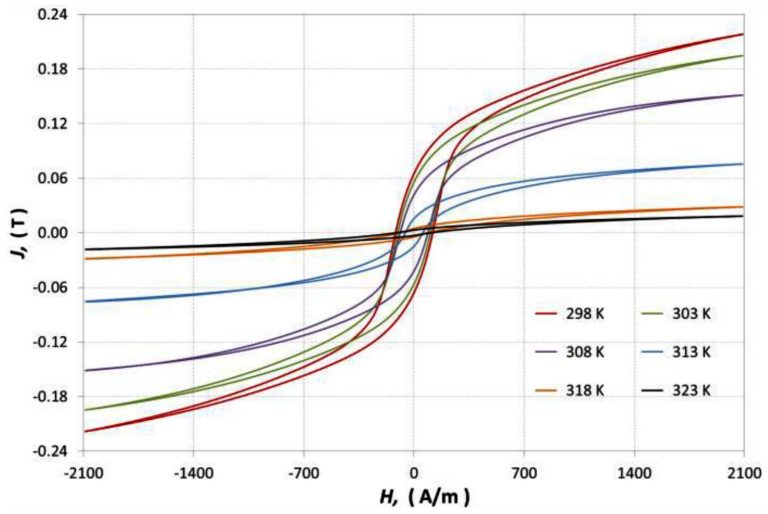

Fig. 2. Temperature dependence of quasi-static hysteresis loops $J(H)$ close to the phase transition $T_{\mathrm{C}}=306 \mathrm{~K}$.

Operation conditions of AMR made of LaFeCoSi composition near the Curie point $T_{\mathrm{C}}$ are most desirable due to maximum magnetic entropy changes. The relatively slow drop of magnetic saturation $J_{S}$ around $T_{\mathrm{C}}$ (Fig. 1) causes magnetic hysteresis as a response to the alternating magnetic field (Fig. 2). Instead of unipolar switching field, experimental investigations were carried out at the bipolar magnetic field to ensure compliance of the measurements with the IEC standards used for ring samples. The magnetic studies show changes in the magnitude of specific power losses $P_{S}$ influenced by frequency, temperature, and magnetic saturation. In Figs. 1 and 2 the impact of temperature on magnetic polarization $J_{m}$ and shape of hysteresis loops is shown.

Shapes of magnetic hysteresis loops $J(H)$ (Fig. 1), $J_{m}(T)$ (Fig. 2), $J_{m}\left(H_{m}\right)$ (Fig. 3) magnetization curves of the bulk sample and the sintered one presented in the previous work [19] are similar.

The transition from the ferromagnetic to the paramagnetic state takes place gradually and the sudden drop of the magnetic polarization is not observed. At the temperature $10 \mathrm{~K}$ above $T_{\mathrm{C}}$ the sample still exhibits ferromagnetic properties (Fig. 1).

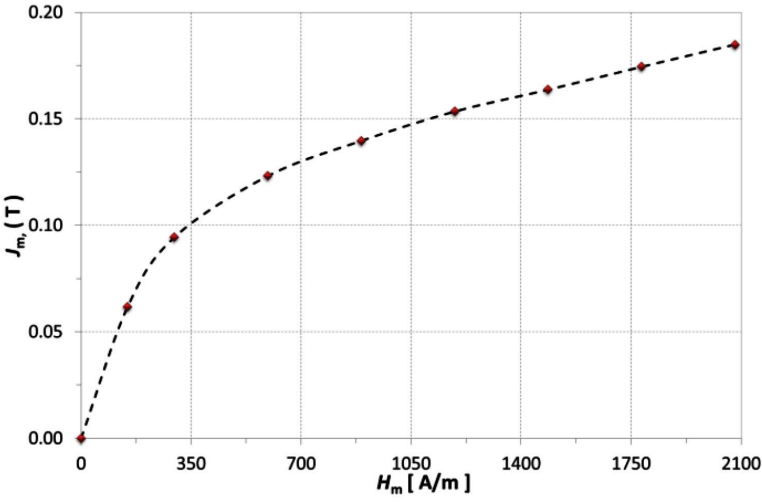

Fig. 3. The normal magnetization curve $J_{m}\left(H_{m}\right)$ recorded at the temperature $T_{\mathrm{C}}=306 \mathrm{~K}$.

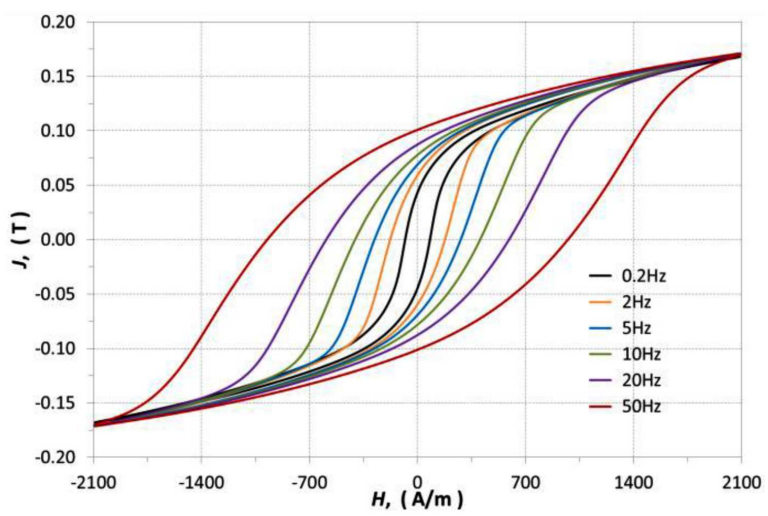

Fig. 4. Impact of magnetic field strength frequency on dynamic hysteresis loops at $T_{\mathrm{C}}=306 \mathrm{~K}$.

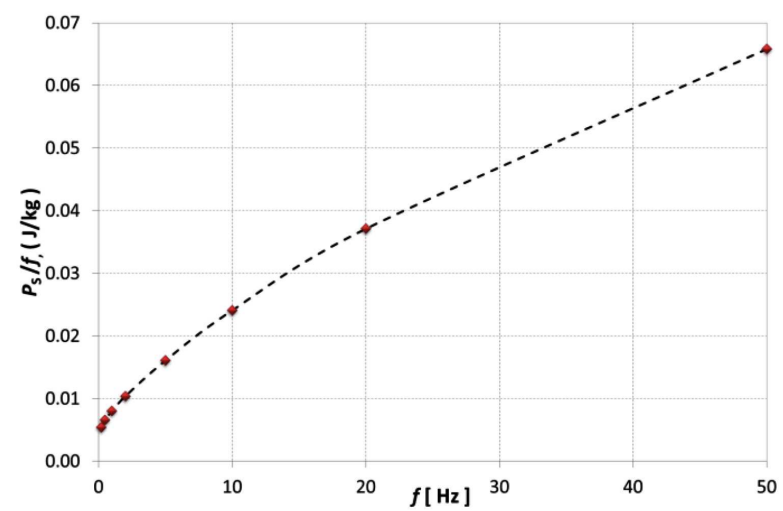

Fig. 5. Calculated energy loss per cycle $P_{S} / f$ as function of frequency.

Measurements of dynamic hysteresis loops (Fig. 4) present a significant rise of energy losses (Fig. 5) in the solid sample. The threshold in the rise of specific power losses $P_{S}$ (Fig. 6) above the frequency of $1 \mathrm{~Hz}$ may be considered as an experimental approximation of the limit where the magnetization process may be considered yet as quasi-static [20]. The growth of the energy losses as 


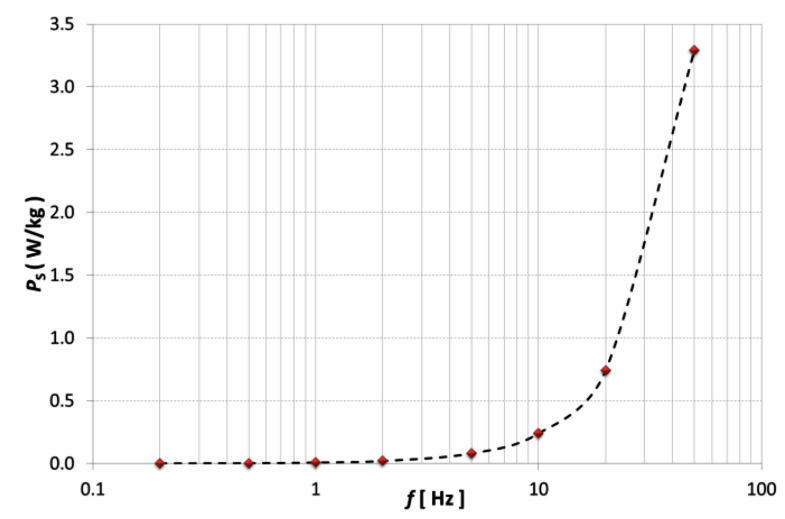

Fig. 6. Frequency dependence of specific power losses $P_{S}$ ranging from $0.2 \mathrm{~Hz}$ to $50 \mathrm{~Hz}$ at the Curie temperature $T_{\mathrm{C}}$.

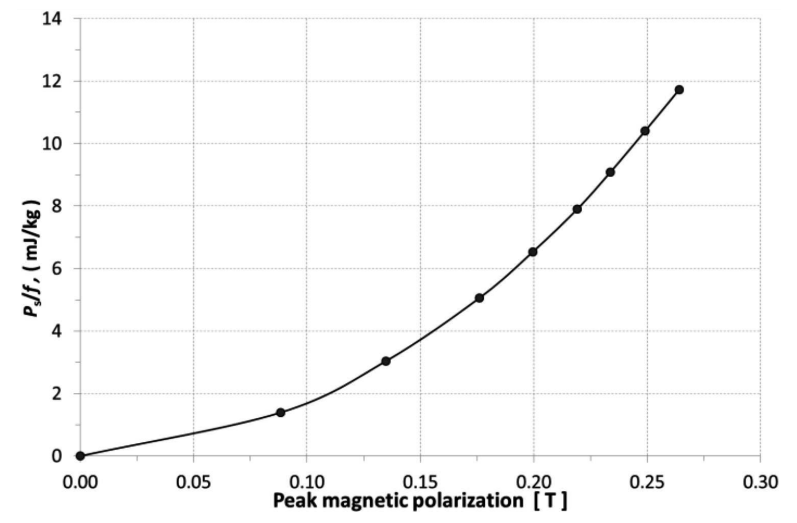

Fig. 7. Influence of peak magnetic polarization on energy loss $P_{S} / f$ at the frequency of $1 \mathrm{~Hz}$ and the temperature $T_{\mathrm{C}}$.

a function of magnetic polarization (Fig. 7) is well described with the exponential Steinmetz equation with the fractional exponent value $\alpha=2.051$ and Steinmetz's coefficient $k_{s}=0.181$. In that case also scaling analysis may be an appropriate tool for detailed study of magnetic power losses [21, 22].

The observed decline of energy losses in Fig. 8 follows the changes of magnetic polarization due to the magnetic phase transition. Nevertheless, the magnitude of dissipated energy at the Curie point is considerable. Taking into account only the interrelation of entropy changes and energy losses, the effective operating temperature range of AMRs ought to be located slightly above $T_{\mathrm{C}}$.

\section{Magnetic power losses balance}

Assuming the Bertotti model of the distribution of energy losses into hysteresis loss $W_{h}$, eddy currents loss $W_{c l}$, and anomalous loss $W_{\text {exc }}$, one can determine the quantitative evaluation of the components in the balance [23]. The decomposition of the total losses into components gives the possibility to identify the sources of these losses [24] and indicates the potential areas for

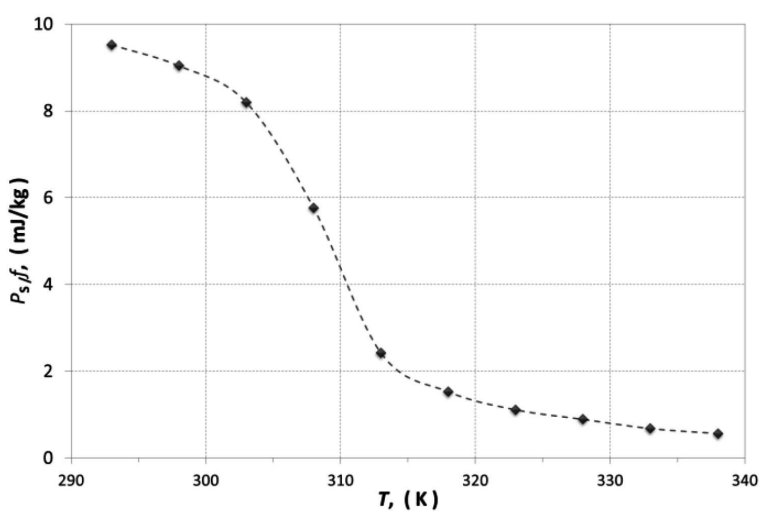

Fig. 8. Impact of temperature and magnetic phase transition on calculated energy loss per cycle $P_{S} / f$ at the frequency of $1 \mathrm{~Hz}$.

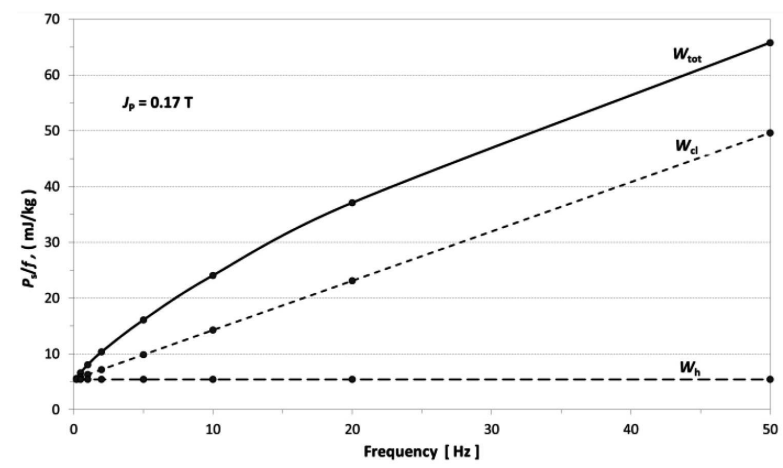

Fig. 9. Energy losses separation in the low-frequency range; peak magnetic polarization $J_{p}=0.17 \mathrm{~T}$.

improving the efficiency of magnetocaloric heat transport systems. Figure 9 depicts the separated components $W_{h}$, $W_{c l}, W_{\text {exc }}$ based on the data from Figs. 4, 5 and numerical data of the components calculated at the frequency of $1 \mathrm{~Hz}$ and temperature $T_{\mathrm{C}}$ are collected in Table I.

The dominant contribution of hysteresis loss component $E_{h}$ results from coercivity and structural properties of the tested sample. Therefore, more detailed X-ray diffractometric study ought to be done. Below frequency of $1 \mathrm{~Hz}, W_{c l}$ component is lower than $W_{\text {exc }}$ although the sample was made of a bulk cast. The nonlinear rise of energy loss per cycle ranging from $0.2 \mathrm{~Hz}$ to $10 \mathrm{~Hz}$ might be explained by a significant contribution of excess loss component in the total loss balance.

Magnitudes of energy losses components at

TABLE I the frequency of $1 \mathrm{~Hz}$ and $T_{\mathrm{C}}$.

\begin{tabular}{c|c}
\hline \hline $\begin{array}{c}\text { Energy loss } \\
\text { per cycle } W=P_{s} / f\end{array}$ & $\begin{array}{c}\text { Magnitude } \\
\text { of energy }[\mathrm{mJ}]\end{array}$ \\
\hline$W_{h}$ & 5.43 \\
$W_{c l}$ & 0.89 \\
$W_{\text {exc }}$ & 1.71 \\
$\left.W_{\text {tot }}\right|_{1 \mathrm{~Hz} ; J_{p}=0.17 \mathrm{~T}}$ & 8.03
\end{tabular}




\section{Summary}

The magnetic studies confirmed a typical ferromagnetic behavior of $\mathrm{LaFe}_{10.92} \mathrm{Co}_{1.08} \mathrm{Si}_{1.2}$ alloy in the lowfrequency bipolar magnetic field. The model of magnetic losses separation did not depend on temperature and was able to determine loss components in the Curie temperature. The contribution of the hysteresis loss component in the energy balance was dominant and confirmed the quasi-static behavior of the magnetization process in the range of frequencies below $1 \mathrm{~Hz}$. Further studies ought to cover the analysis of magnetic properties and power losses under the DC-biased magnetic field which is more compliant with real operation conditions of magnetocaloric AMR.

\section{Acknowledgments}

The work was carried out with financial support from Polish National Science Centre under the grant agreement $6370 / \mathrm{B} / \mathrm{T} 02 / 2011 / 40$.

\section{References}

[1] A.M. Tishin, Y.I. Spichkin, The Magnetocaloric Effect and Its Applications, IOP Publ., Bristol 2003.

[2] V. Franco, J.S. Blázquez, J.J. Ipus, J.Y. Law, L.M. Moreno-Ramírez, A. Conde, Prog. Mater. Sci. 93, 112 (2018).

[3] J.S. Brown, P.A. Domanski, J. Appl. Thermal Eng. 64, 252 (2014)

[4] V.K. Pecharsky, K.A. Gschneidner Jr., Phys. Rev. Lett. 78, 4494 (1997)

[5] F.C. Nascimento, A. Oliveira dos Santos, A.D. Campos, S. Gama, L.P. Cardoso, Mater. Res. 9, 111 (2006).

[6] J. Ferenc, M. Kowalczyk, R. Wróblewski, G. Cieślak, K. Sielicki, M. Leonowicz, T. Kulik, Acta Phys. Pol. A 128, 111 (2015).
[7] J.Y. Law, V. Franco, R.V. Ramanujan, J. Appl. Phys. 111, 113919 (2012).

[8] Z. Śniadecki, J. Marcin, I. Škorvánek, N. Pierunek, B. Idzikowski, J. Alloys Comp. 584, 477 (2014).

[9] T.T.M. Palstra, J.A. Mydosh, G.J. Nieuwenhuys, A.M.V.D. Kraan, K.H.J. Buschow, J. Magn. Magn. Mater. 36, 290 (1983).

[10] X.B. Liu, Z. Altounian, J. Magn. Magn. Mater. 264, 209 (2003).

[11] P. Gębara, P. Pawlik, B. Michalski, J.J. Wysłocki, K. Kotynia, Acta Phys. Pol. A 128, 87 (2015).

[12] P. Gębara, P. Pawlik, B. Michalski, J.J. Wysłocki, Acta Phys. Pol. A 127, 576 (2015).

[13] A. Yan, K.H. Muller, O. Gutfleisch, J. Alloys Comp. 450, 18 (2008).

[14] P. Gębara, P. Pawlik, I. Skorvanek, J. Marcin, J.J. Wysłocki, Acta Phys. Pol. A 118, 910 (2010).

[15] V.K. Pecharsky, K.A. Gschneidner Jr, J. Magn. Magn. Mater. 200, 44 (1999).

[16] R. Gozdur, K. Chwastek, M. Najgebauer, M. Lebioda, Ł. Bernacki, A. Wodzynski, Acta Phys. Pol. A 131, 801 (2017).

[17] R. Gozdur, P. Gębara, K. Chwastek, Open Phys. 16, 266 (2018).

[18] R. Gozdur, A. Majocha, Przegl. Elektrotech. 86, 79, (2010) (in Polish).

[19] R. Gozdur, M. Lebioda, L. Bernacki, Acta Phys. Pol. A 128, 98 (2015).

[20] R. Gozdur, A. Majocha, Przegl. Elektrotech. 83, 134 (2007) (in Polish).

[21] M. Najgebauer, Acta Phys. Pol. A 128, 107 (2015).

[22] R. Gozdur, M. Najgebauer, J. Electr. Eng. 66, 37 (2015).

[23] G. Bertotti, IEEE Trans. Magn. 24, 621 (1988).

[24] G. Bertotti, J. Magn. Magn. Mater. 320, 2436 (2008). 